\section{Electrochemical current rectifier as a highly sensitive and selective cytosensor for cancer cell detection $\dagger$}

\author{
Hailong Li, ${ }^{a b}$ Dan $\mathrm{Li}^{a}{ }^{a}$ Jiyang Liu, ${ }^{a b}$ Yinan Qin, ${ }^{a}$ Jiangtao Ren, ${ }^{a b}$ Shanling Xu, ${ }^{a}$ \\ Yaqing Liu, ${ }^{* a}$ Dirk Mayer ${ }^{* c}$ and Erkang Wang ${ }^{* a}$
}

Received 5th December 2011, Accepted 6th January 2012

DOI: $10.1039 / \mathbf{c} 2 \mathrm{cc17591f}$

Signal amplification originating from electrochemical current rectifier (ECR) was firstly applied to construct a cytosensor for rapid and non-invasive detection of folate receptor-rich cancer cells with high sensitivity. It exhibits a broad linear range with a detection limit as low as 10 cells $\mathrm{mL}^{-1}$ even in the presence of a large number of normal cells.

Cancer is considered as a worldwide mortal sickness and has become a major public concern nowadays. It is highly desirable to develop rapid and simple method to detect cancer cells, which would make a huge impact on preclinical diagnosis and reduction in mortality for certain cancers. To date, various methods have been developed for cancer cell detection, including cytological testing, fluorescent imaging, magnetic resonance imaging, positron emission tomography, computerized tomography, X-ray, radiography and ultrasound. ${ }^{1,2}$ However, most of these modalities are costly in the experimental process or instrumentation. Moreover, those approaches may be coupled with radioactive risk. Therefore new techniques for cancer cell detection are urgently demanded. Recently, simple and nondestructive electrochemical methods have attracted considerable attention for developing cell-based biosensors. Various methods were explored to improve detection sensitivity. ${ }^{3-5}$ Herein, we provided a simple and novel approach to detect HeLa cells with high sensitivity and selectivity by combining a molecular device, an electrochemical current rectifier (ECR).

As a basic molecular device, ECR only permits unidirectional current to pass through and has been extensively investigated since the first report. ${ }^{6}$ To successfully fabricate an ECR, direct electron transfer between a solution-phase redox probe and the underlying electrode should be insulated. ${ }^{6}$ For monolayerbased ECRs, redox-active electron transfer (ET) mediators are usually immobilized on the electrode surface with an insulating

${ }^{a}$ State Key Lab of Electroanalytical Chemistry, Changchun Institute of Applied Chemistry, Chinese Academy of Sciences, Changchun, 130022.E-mail:yaqingliu@ciac.jl.cn,ekwang@ciac.jl.cn;

Fax: (+86) 431 85689711; Tel: $(+86) 43185262003$

${ }^{b}$ Graduate School of the Chinese Academy of Sciences, Beijing 100039, China

Institute of Complex Systems Bioelectronics, Peter Gruenberg Institute, Research Center Juelich, 52425, Germany.

E-mail:dirk.mayer@fz-juelich.de

$\dagger$ Electronic supplementary information (ESI) available: Experimental section and supplementary figures. See DOI: 10.1039/c2cc17591f layer and present a current signal with low magnitude in the supporting electrolyte, as shown in Scheme 1A. The surfaceconfined redox probes function as ET mediators to modulate electrochemical behavior of the solution-phase redox probe. According to the relative redox potential between ET mediator and redox probe, only a unidirectional current signal from the solution-phase redox probe with higher magnitude is allowed to pass through, as shown in Scheme 1B. The signal amplification may foster the development of ECRs in constructing sensitive biosensors. Current response is decreased upon binding cells on the modified electrode since electron transfer access was inhibited by the insulating cell membrane, as shown in Scheme 1C. In this Communication, as a proof of concept, we demonstrated the application of ECR-based signal amplification in selective detection of human cervical carcinoma (HeLa) cells for the first time.

Here, the ECR was prepared by immersing a gold bead electrode into a mixture of 11-undecanethiol-1-ferrocene (UDT-Fc) and 16-mercaptohexadecanoic acid (MHDA), which function

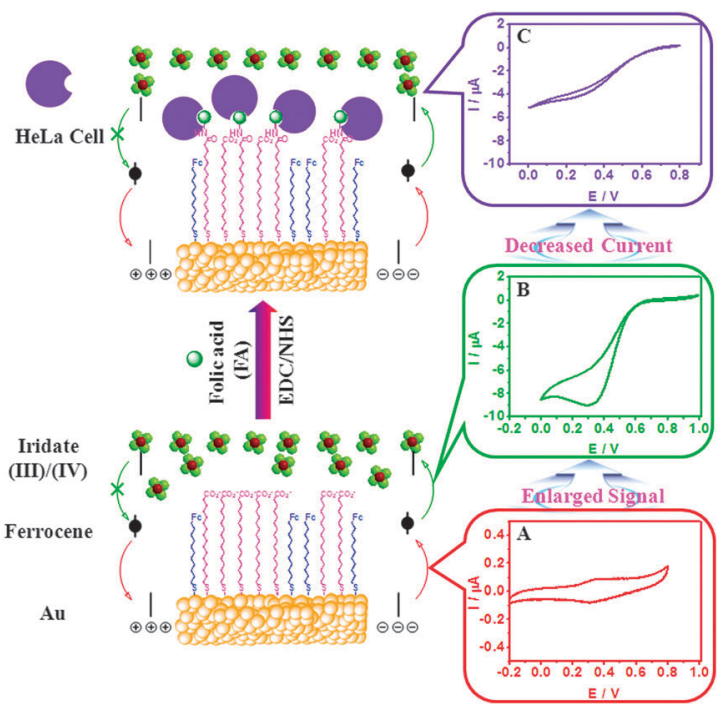

Scheme 1 Electron-transfer mechanism of cathodic electrochemical current rectification and the resultant signal amplification in the present system (from A to B). Folic acid was covalently immobilized to the electrode to target folate receptor-rich HeLa cells. A decreased signal was produced upon cell binding (from $\mathrm{B}$ to $\mathrm{C}$ ). 


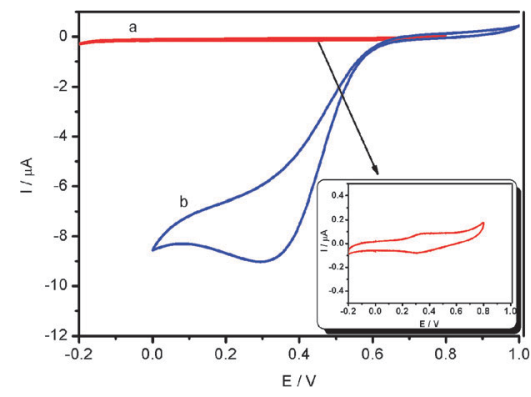

Fig. 1 Cyclic voltammograms of $\mathrm{Au} /(\mathrm{UDT}-\mathrm{Fc})-\mathrm{MHDA}$ in PBS (a) and $5 \mathrm{mM} \mathrm{K}_{2} \mathrm{IrCl}_{6}$ solution (b), respectively. Inset is the magnified image of (a). Scan rate: $50 \mathrm{mV} \mathrm{s}^{-1}$.

as ET mediator and insulating layer, respectively, resulting in a modified electrode $\mathrm{Au} /(\mathrm{UDT}-\mathrm{Fc})-\mathrm{MHDA}$. As shown in Fig. 1a, a pair of redox peaks originating from ferrocene were monitored in PBS buffer, confirming the successful immobilization of UDT-Fc on the gold electrode. The redox potential was found to be $0.35 \mathrm{~V}$. The observed redox current was low due to the small amount of redox-active molecules on the surface. After addition of $\mathrm{K}_{2} \mathrm{IrCl}_{6}$ into the solution, only cathodic, and no anodic, current was detected, as shown in Fig. $1 \mathrm{~b}$, which is a feature of ECR. ${ }^{6}$ Under the circumstances, the ET between solution-phase redox probes and the electrode was mediated by the surface-confined ferrocenylthiol while inhibited by the MHDA monolayer. The inhibition of MHDA was confirmed by the fact that neither redox peaks nor current rectification behaviour related to $\mathrm{K}_{2} \mathrm{IrCl}_{6}$ was observed at the $\mathrm{Au} / \mathrm{MHDA}$ electrode (Fig. S1 in ESI $\uparrow$ ), which is similar to our previous finding. ${ }^{7}$ The electron transfer between the solutionphase redox probe and the underlying electrode could only be accessed at the sites where mediators were immobilized. During the negative potential sweep, the ferrocene received electrons from the gold electrode as soon as the reduction potential of ferrocene was reached. Considering the relative redox potentials of surface-confined ferrocene $(0.34 \mathrm{~V})$ and iridate(IV/III) ions $(0.71 \mathrm{~V}),{ }^{6 c}$ the electrons were forwarded immediately to the iridate(IV) ions, leading to an enhanced cathodic current. In the reverse sweep, however, the electron transfer between the oxidized ferrocene and iridate(III) ions was thermodynamically forbidden, resulting in the absence of anodic current. The modified electrode can be referred to as a cathodic ECR. Obviously, the current signal of the as-prepared electrode in supporting electrolyte was strongly enhanced (about 50 times) after addition of redox probes into the system, which is consistent with previous reports. ${ }^{6 c}$ This kind of signal amplification can be used to construct sensitive cytosensors for cancer cells. For further cancer cell detection, the stability of the as-prepared electrode was evaluated by recording current responses at different time. No obvious change was observed (Fig. S2 in ESI $\dagger$ ), indicating the as-prepared ECR was qualified to be a biosensor.

In our investigations, HeLa cells were used as a model to develop cytosensors. It was known that folate receptor (FR) is overexpressed on the membrane of HeLa cell, which is a common feature of epithelial cancers. ${ }^{5}$ Folic acid (FA) is a kind of vitamin and is essential to cell survival and proliferation. It presents higher affinity to FRs and is used for target delivering cargos of many sorts, such as imaging agents, proteins, liposomes, chemotherapeutics, and so on. ${ }^{8}$ Therefore, FA was covalently immobilized on the MHDA layer of the as-prepared ECR through an amide bond to selectively detect the HeLa cells. Upon the binding of FA to MHDA, the current response was decreased due to the insulating of FA (Fig. S3 in ESI $\dagger$ ). Too low a FA density decreases the sensor sensitivity while too much will lead to a greatly decreased current response. The optimal density of immobilized FA was achieved by controlling the reaction time. Herein, the as-prepared FA-modified electrode was used as cytosensor. The basic idea of cell-based sensors is that the access to electron transfer would be hindered after immobilization of cells on the electrode surface due to the resistance of the cell membrane. ${ }^{4}$ The current response would be different if the as-prepared sensors were immersing in HeLa cell suspension $\left(10^{5}\right.$ cells $\left.\mathrm{mL}^{-1}\right)$ for a different length of time (Fig. S4 in ESI $\dagger$ ). The peak current significantly decreased after incubation in the cell solution $10 \mathrm{~min}$ and then no obvious change was observed until an incubation time of $30 \mathrm{~min}$. The time needed to reach a plateau is related to the cell concentration, which takes a longer time when immersing in a suspension with lower concentration and a shorter time when immersing in a suspension with higher concentration. To keep the same experimental conditions, $10 \mathrm{~min}$ was chosen as the optimal incubation time for HeLa cell detection in the sensing system. This incubation time is much shorter in comparison with classical molecular biology methods with radioactive and optical labels (hours to days), greatly avoiding the possibility of contamination by microbes during measurement. ${ }^{1}$

To reduce the loss of cell viability, differential pulse voltammetry (DPV) was performed to detect living cells since the effects of long exposure to an electrical field on the cells could be avoided with DPV measurement. ${ }^{9}$ The degree of the current decrease upon HeLa cell attachment depends on the amount of cells seeded on the sensing electrode, which is related to the cell concentration within the same incubation time. Fig. 2A shows the DPV responses of the FA-modified electrode after incubation in HeLa suspensions with different concentrations. The adsorption of cells obeys a Langmuir function. As the HeLa concentration increased, the current changes reached a plateau because the electrode surface gets crowded and the cells have to compete for a free spot to bind to (Fig. S5 in $\mathrm{ESI} \dagger)$. The peak current intensity linearly decreased with the
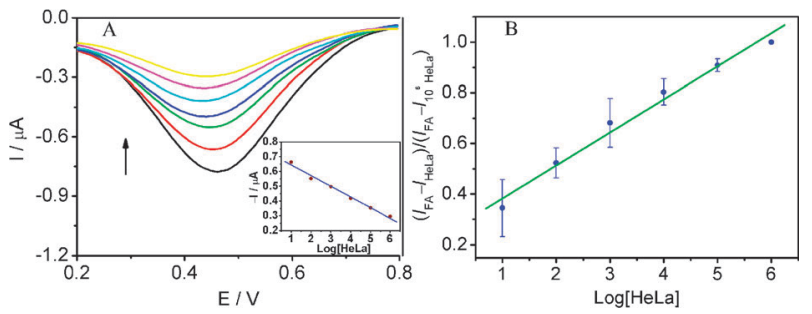

Fig. 2 (A) DPV responses of the FA-modified electrode after being incubated with different concentrations of HeLa cells in saline: 0, 10, $10^{2}, 10^{3}, 10^{4}, 10^{5}, 10^{6}$ cells $\mathrm{mL}^{-1}$ (from bottom to top). Inset shows the linear relationship between the peak current intensity and the logarithm of HeLa cell concentration. (B) The current change against the logarithm of HeLa cell concentration with error bars. 
logarithm of HeLa cells concentration as shown in the inset of Fig. 2A. To evaluate the repeatability of the biosensors, error bars were calculated by plotting the degree of current decrease $\left(I_{\mathrm{Blank}}-I_{\mathrm{HeLa}}\right) /\left(I_{\mathrm{Blank}}-I_{10^{6} \mathrm{HeLa}}\right)$ against the logarithm of $\mathrm{HeLa}$ concentration, where $I_{\mathrm{Blank}}, I_{\mathrm{HeLa}}$ and $I_{10^{6} \mathrm{HeLa}}$ are the current intensities in the absence of cells, different concentrations of HeLa cells and $10^{6} \mathrm{HeLa}$ cells $\mathrm{mL}^{-1}$, respectively. Fig. 2B shows the linear relationship of signal changes as a function of the logarithm of HeLa cell concentration from 10 to $10^{6}$ cells $\mathrm{mL}^{-1}\left(R^{2}=0.99\right)$, indicating good detection repeatability. The as-prepared sensors exhibited a high sensitivity, and a detection limit as low as 10 cells $\mathrm{mL}^{-1}$ was reached.

To further evaluate the selectivity of the present system, DPV was also used to monitor HeLa cells in the presence of normal cells HEK $293\left(10^{5}\right.$ cells $\left.\mathrm{mL}^{-1}\right)$. It is known that alpha isoform of the folate receptors could work as tumor indicator, which are usually overexpressed on many epithelial cancers while limitedly expressed on normal tissues. ${ }^{5,10}$ Therefore, it was expected that the as-prepared sensors could selectively detect HeLa cells in the presence of normal cell according to the special recognition between FR and FA (Fig. S6 in ESI $\dagger$ ). Fig. 3A shows the DPV responses of the sensors on HeLa cells at different concentrations in the presence of HEK 293. The peak current intensity linearly decreased with the logarithm of HeLa cell concentrations as shown in the inset of Fig. 3A. The degree of current decrease $\left(I_{\mathrm{Blank}}-I_{\mathrm{HeLa}}\right) /\left(I_{\mathrm{Blank}}-I_{10^{6} \mathrm{HeLa}}\right)$ linearly increases against logarithm of HeLa cell concentration in the range from 0 to $10^{6} \mathrm{HeLa}$ cells $\mathrm{mL}^{-1}\left(R^{2}=0.98\right)$, as shown in Fig. 3B. The response to HeLa cell concentrations as low as 10 cells $\mathrm{mL}^{-1}$ was obvious even in the presence of a large number of normal cells $\left(10^{5}\right.$ cells $\mathrm{mL}^{-1}$ HEK 293), confirming the high selectivity as well as high sensitivity of the provided method. The detection limit is equal to that reported by Wang et al. and much lower than those reported by other groups. ${ }^{5,11}$ Wang et al. obtained a detection range from 10 to $10^{5}$ cells $\mathrm{mL}^{-1}$ with an AC impedimetric approach. ${ }^{7}$ Here, the as-prepared sensors show a wider detection range with DPV and the detection could be finished quickly within half a minute, greatly reducing the effect of the electric field on the cells.

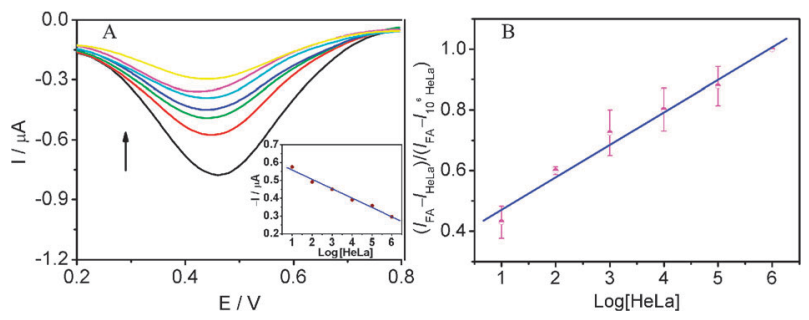

Fig. 3 (A) DPV responses of the FA-modified electrode after being incubated with different concentrations of HeLa cells in the presence of $10^{5}$ cells $\mathrm{mL}^{-1}$ HEK 293 (from bottom to top: 0, 10, 10 $2,10^{3}, 10^{4}$, $10^{5}, 10^{6}$ cells $\mathrm{mL}^{-1} \mathrm{HeLa}$ ). Inset shows the linear relationship between the peak current intensity and the logarithm of HeLa cells concentration. (B) The corresponding current change against the logarithm of $\mathrm{HeLa}$ cells concentration with error bars.
In conclusion, we demonstrated the proof of concept that the signal amplification originating from ECR can be applied in FR-rich cancer cell detection for the first time. The surface conjugated FA plays an important role in the selective detection of HeLa cells. A wide detection range and low detection limit of 10 cells $\mathrm{mL}^{-1}$ was obtained even in the presence of a large number of normal cells (HEK 293), which was ascribed to the current magnification from the as-prepared ECR. The explored system has great advantages for the development of cytosensors. Firstly, the present approach is simple and reliable. The developed cytosensor is easy to operate and noninvasive on cell detection, exhibiting good repeatability as well as high sensitivity and selectivity. Secondly, the detection was performed with a fast-response DPV method, which can avoid the effects of long exposure to an electric field on the immobilized cells and reduce the loss of cell viability. Last, but not least, the developed ECR-based cytosensor expands the application of molecular devices and exhibits great potential for application in the development of biosensors.

This work was supported by National Natural Science Foundation of China (No. 21190040, 21075120 and 21105095) and 973 project (No. 2010CB933600 and 2009CB930100).

\section{Notes and references}

1 (a) K. Brindle, Nat. Rev. Cancer, 2008, 8, 94; (b) T. Mosmann, J. Immunol. Methods, 1983, 65, 55; (c) J. Zhang, R. E. Campbell, A. Y. Ting and R. Y. Tsien, Nat. Rev. Mol. Cell Biol., 2002, 3, 906.

2 L. Fass, Mol. Oncol., 2008, $2,115$.

3 (a) I. Giaever and C. R. Keese, Nature, 1993, 366, 591; (b) J. Liu, H. Zhou, J. Xu and H. Y. Chen, Chem. Commun., 2011, 47, 4388; (c) P. Seriburi, S. McGuire, A. Shastry, K. F. Bohringer and D. R. Meldruim, Anal. Chem., 2008, 80, 3677.

4 J. Hong, K. Kandasamy, M. Marimuthu, C. S. Choi and S. Kim, Analyst, 2011, 136, 237.

5 J. Weng, Z. Zhang, L. Sun and J. A. Wang, Biosens. Bioelectron., 2011, 26, 1847.

6 (a) G. P. Kissling, C. Buenzli and D. J. Fermin, J. Am. Chem. Soc., 2010, 132, 16855; (b) Y. Liu, A. Offenhaeusser and D. Mayer, Biosens. Bioelectron., 2010, 25, 1173; (c) Y. Liu, A. Offenhaeusser and D. Mayer, Angew. Chem., Int. Ed., 2010, 49, 2595; (d) H. D. Abruna, P. Denisevich, M. Umana, T. J. Meyer and R. W. Murray, J. Am. Chem. Soc., 1981, 103, 1; (e) K. S. Alleman, K. Weber and S. E. Creager, J. Phys. Chem., 1996, 100, 17050; (f) P. G. Pickup, W. Kutner, C. R. Leidner and R. W. Murray, J. Am. Chem. Soc., 1984, 106, 1991; (g) D. T. Rong, H. G. Hong, Y. I. Kim, J. S. Krueger, J. E. Mayer and T. E. Mallouk, Coord. Chem. Rev., 1990, 97, 237; (h) Y. Liu, A. Offenhaeusser and D. Mayer, Bioelectrochemistry, 2010, 77, 89; (i) S.-K. Oh, L. A. Baker and R. M. Crooks, Langmuir, 2002, 18, 6981.

7 Y. Qin, L. Xu, J. Ren, Y. Liu and E. Wang, Chem. Commun., 2011, 47, 8232 .

8 (a) N. Kohler, C. Sun, J. Wang and M. Q. Zhang, Langmuir, 2005, 21, 8858; (b) A. R. Hilgenbrink and P. S. Low, J. Pharm. Sci., 2005, 94, 2135 .

9 M.-L. Shao, H.-J. Bai, H.-L. Gou, J.-J. Xu and H.-Y. Chen, Langmuir, 2009, 25, 3089.

10 (a) I. G. Campbell, T. A. Jones, W. D. Foulkes and J. Trowsdale, Cancer Research, 1991, 51, 5329; (b) L. R. Coney, A. Tomassetti, L. Carayannopoulos, V. Frasca, B. A. Kamen, M. I. Colnaghi and V. R. Zurawski, Cancer Research, 1991, 51, 6125.

11 (a) W. Cheng, L. Ding, J. Lei, S. Ding and H. Ju, Anal. Chem., 2008, 80, 3867; (b) C. Hao, L. Ding, X. Zhang and H. Ju, Anal. Chem., 2007, 79, 4442; (c) Z. Shen, M. Huang, C. Xiao, Y. Zhang, X. Zeng and P. G. Wang, Anal. Chem., 2007, 79, 2312; (d) C. M. Ruan, L. J. Yang and Y. B. Li, Anal. Chem., 2002, 74, 4814. 